\title{
Development of Computational 3D MoM Algorithm for Nanoplasmonics
}

\author{
Nadson W. P. de Souza, Karlo Q. da Costa, Victor Dmitriev \\ Federal University of Para,Belém-PA, Brazil, e-mails: nadsonwelkson@hotmail.com,karlo@ufpa.br, \\ victor@ufpa.br
}

\begin{abstract}
In this paper, we present an algorithm for full-wave electromagnetic analysis of nanoplasmonic structures. We use the three-dimensional Method of Moments to solve the electric field integral equation. The computational algorithm is developed in the language $C$. As examples of application of the code, the problems of scattering from a nanosphere and a rectangular nanorod are analyzed. The calculated characteristics are the near field distribution and the spectral response of these nanoparticles. The convergence of the method for different discretization sizes is also discussed.
\end{abstract}

Index Terms-Plasmonics, metal nanoparticles, optical scattering, spectral response, method of moments.

\section{INTRODUCTION}

Nanoplasmonics studies interaction of optical fields with metallic nanostructures beyond the diffraction limit of light. At optical frequencies, metals exhibit electrical charge oscillations known as plasmons or surface plasmon resonances [1]-[3]. The resonances of these electrical oscillations depend on the electrical properties of the metal element, on its dimensions and geometry and on polarization of the incident electromagnetic wave. In literature, one can find analysis of plasmonic nanoparticles with different geometries, such as spheres, rods [11], stars [12], nanoburguer [13], tetrahedral [14], circular [15] and triangular nanodisks [16], etc. The growing number of publications in this area in recent years can be explained by numerous possible applications such as superresolution microscopy [4], nanoantennas for nanophotonics [5]-[9], ultra-high-density optical data storage devices [10], among others.

In this paper, we present a computational algorithm developed for nanoplasmonics which is based on three-dimensional Method of Moments (3D MoM) [17]. Using this algorithm, we analyze electromagnetic scattering of optical fields by metallic nanoparticles with different geometries. To characterize the complex dielectric constant of gold nanoparticles in optical frequencies, we use in the analysis the Lorentz-Drude model. The computational implementation of the method is performed using the language $\mathrm{C}$. As examples of application of the code, two problems of scattering of a nanosphere and a rectangular nanorod are resolved. We calculate distribution of the near field, the spectral response and analyze the resonances of these particles. The convergence of the method for different discretization sizes is also investigated. To validate the developed code, we compare our 
results with simulations carried out by the commercial package Comsol Multiphysics and by the analytical Mie model for spherical particle.

\section{THEORY}

\section{A. Description of the Problem}

In Fig. 1, the geometries considered in this paper are shown. The problems consist of the electromagnetic scattering from a single nanosphere (Fig. 1a) and from a rectangular nanorod (Fig. 1b) made of gold centered at the origin of the rectangular coordinate system in free space.

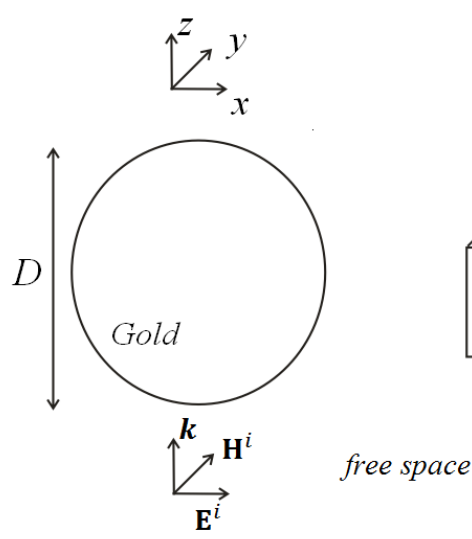

(a)

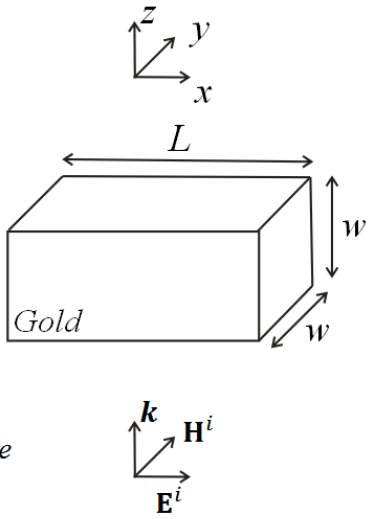

(b)

Fig.1. Geometries of the analyzed electromagnetic scattering problems: (a) nanosphere, (b) rectangular nanorod.

The nanosphere has the diameter $\mathrm{D}=2 \mathrm{a}=120 \mathrm{~nm}$ and the rectangular nanorod has the length $\mathrm{L}=60$ $\mathrm{nm}$ and the square cross section with the dimension $\mathrm{w}=10 \mathrm{~nm}$. Each structure is illuminated by an incident plane wave $\mathbf{E}^{\mathrm{i}}=E_{i 0} e^{-j \boldsymbol{k} \cdot \boldsymbol{r}} \boldsymbol{a}_{x}$ with the time dependence $e^{j \omega t}$, where $\omega$ is the angular frequency, $\boldsymbol{k}$ the wave vector, $\boldsymbol{r}$ is the position vector and $E_{i 0}=1 \mathrm{~V} / \mathrm{m}$ the amplitude of the incident wave. Nanoparticles are described by complex permittivity $\varepsilon=\varepsilon_{r} \varepsilon_{0}$, where $\varepsilon_{0}$ is the permittivity of free space and the $\varepsilon_{r}$ the relative permittivity which can be approximated by the Lorentz-Drude model for the gold with one interband term as follows:

$$
\varepsilon_{r}=\varepsilon_{\infty}-\frac{\omega_{p 1}^{2}}{\omega^{2}-j \Gamma \omega}+\frac{\omega_{p 2}^{2}}{\omega_{0}^{2}-\omega^{2}+j \gamma \omega}
$$

where $\varepsilon_{\infty}=7, \omega_{p 1}=13.8 \times 10^{15} s^{-1}, \omega_{p 2}=45 \times 10^{14} s^{-1}, \omega_{0}=2 \pi c / \lambda_{0}, \quad \lambda_{0}=450 \mathrm{~nm}, \gamma=9 \times 10^{14} s^{-1}$, $\Gamma=1.075 \times 10^{14} s^{-1}, c$ is the speed of light, and $j$ is the imaginary unit. This model is a good approximation for the complex permittivity at wavelengths greater than $500 \mathrm{~nm}$. It characterizes the dispersion of metals at optical frequencies [2]. 


\section{B. Integral Equation}

The general electromagnetic scattering problem is presented in Fig. 2a. The total electric field $\mathbf{E}$ outside the volume $V_{o b j}$ of the nanoparticle is given by the sum of the incident plane wave and the scattered from the nanoparticle wave:

$$
\mathbf{E}(\boldsymbol{r})=\mathbf{E}^{\mathrm{i}}(\boldsymbol{r})+\mathbf{E}^{\mathrm{s}}(\boldsymbol{r})
$$

where superscripts $\mathrm{i}$ and $\mathrm{s}$ indicate the incident and scattered fields. The latter can be viewed as the field radiated by an equivalent polarization current density $\mathbf{J}_{\mathbf{e q}}\left(\boldsymbol{r}^{\prime}\right)$, with $\boldsymbol{r}^{\prime} \in V_{o b j}$, as shown in Fig. 2b. The scattered fields obey the Maxwell's equations [19]:

$$
\begin{gathered}
\nabla \times \mathbf{E}^{\mathrm{s}}(\boldsymbol{r})=-j \omega \mu_{0} \mathbf{H}^{\mathrm{s}}(\boldsymbol{r}) \\
\nabla \times \mathbf{H}^{\mathrm{s}}(\boldsymbol{r})=j \omega \varepsilon_{0} \mathbf{E}^{\mathrm{s}}(\boldsymbol{r})+\mathbf{J}_{\text {eq }}(\boldsymbol{r}) .
\end{gathered}
$$

The equivalent polarization current density $\mathbf{J}_{\mathbf{e q}}\left(\boldsymbol{r}^{\prime}\right)$ exists only inside the material, and it is given by

$$
\mathbf{J}_{\mathrm{eq}}\left(\boldsymbol{r}^{\prime}\right)=j \omega\left[\varepsilon(\boldsymbol{r})-\varepsilon_{0}\right] \mathbf{E}\left(\boldsymbol{r}^{\prime}\right)=\tau(\boldsymbol{r}) \mathbf{E}\left(\boldsymbol{r}^{\prime}\right),
$$

where $\boldsymbol{r}^{\prime}$ is the vector that indicates the source point, and $\varepsilon(\boldsymbol{r})=\varepsilon_{0}$ if $\boldsymbol{r} \notin V_{o b j}$ and $\varepsilon(\boldsymbol{r})=\varepsilon_{o b j}$ if $\boldsymbol{r} \in$ $V_{o b j}$. From(3) and (4) we obtain the wave equation

$$
\nabla \times \nabla \times \mathbf{E}^{s}(\boldsymbol{r})-k_{0}^{2} \mathbf{E}^{s}(\boldsymbol{r})=-j \omega \mu_{0} \mathbf{J}_{\mathrm{eq}}\left(\boldsymbol{r}^{\prime}\right)
$$

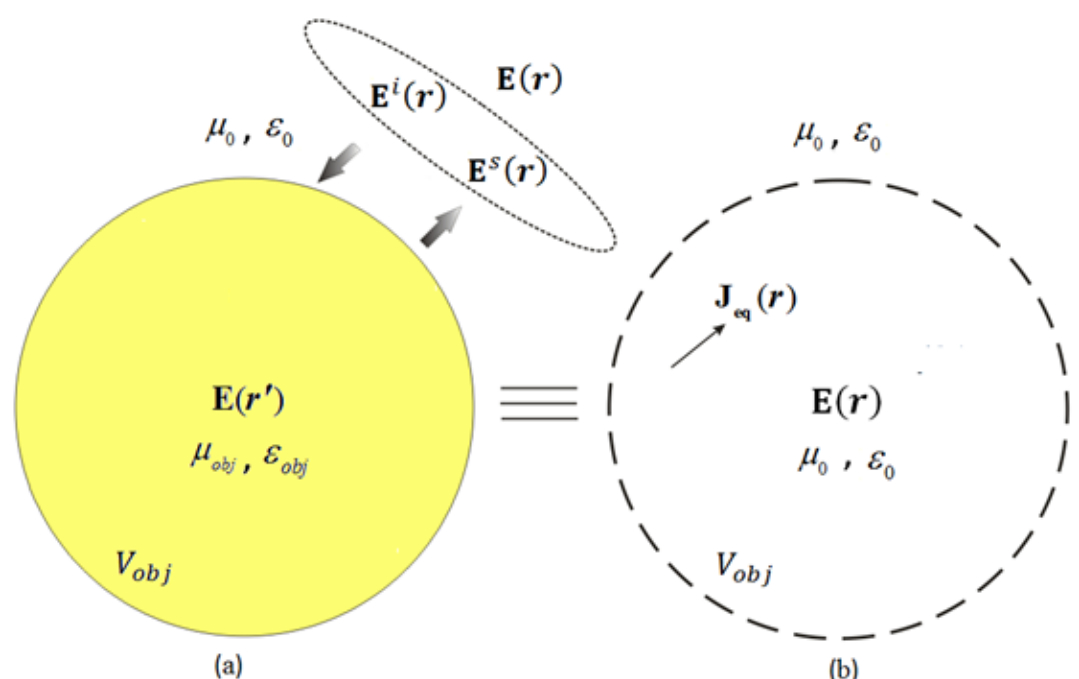

Fig. 2.Geometry of general electromagnetic scattering problem: (a) original problem, (b) equivalent problem. 
where $k_{o}=\omega \sqrt{\mu_{0} \varepsilon_{0}}=2 \pi / \lambda$ and $\lambda$ the wavelength. The solution of (6) is given by

$$
\mathbf{E}^{\mathrm{s}}(\boldsymbol{r})=-j \omega \mu_{0} \int_{V_{o b j}} \overline{\mathbf{G}}_{\mathbf{0}}\left(\boldsymbol{r}, \boldsymbol{r}^{\prime}\right) \cdot \mathbf{J}_{\mathrm{eq}}\left(\boldsymbol{r}^{\prime}\right) d v^{\prime}
$$

$\overline{\mathbf{G}}_{0}$ is the dyadic Green's function for free space defined by

$$
\begin{gathered}
\overline{\mathbf{G}}_{0}\left(\boldsymbol{r}, \boldsymbol{r}^{\prime}\right)=\left(\bar{I}+\frac{1}{k_{0}^{2}} \nabla \nabla\right) g_{0}\left(\boldsymbol{r}, \boldsymbol{r}^{\prime}\right) \\
g_{0}\left(\boldsymbol{r}, \boldsymbol{r}^{\prime}\right)=\frac{\mathrm{e}^{-j k_{0}\left|\boldsymbol{r}-\boldsymbol{r}^{\prime}\right|}}{4 \pi\left|\boldsymbol{r}-\boldsymbol{r}^{\prime}\right|}
\end{gathered}
$$

Equation (7) is used to calculate the scattered field outside the volume $V_{o b j}$ of the nanoparticle. However, to calculate the scattered field inside the nanoparticle, where there is a singularity, one should modify (7) according to [20]

$$
\mathbf{E}^{\mathrm{s}}(\boldsymbol{r})=P V \int_{V_{o b j}} \overline{\mathbf{G}}\left(\boldsymbol{r}, \boldsymbol{r}^{\prime}\right) \cdot \mathbf{J}_{\text {eq }}\left(\boldsymbol{r}^{\prime}\right) d v^{\prime}-\frac{\mathbf{J}_{\text {eq }}(\boldsymbol{r})}{j 3 \omega \varepsilon_{0}}
$$

where $\overline{\mathbf{G}}\left(\boldsymbol{r}, \boldsymbol{r}^{\prime}\right)=-j \omega \mu_{0} \overline{\mathbf{G}}_{0}\left(\boldsymbol{r}, \boldsymbol{r}^{\prime}\right), P V$ means the principal value of the integral and the second term is a correction factor. Substituting (10) in (2), we obtain the following integral equation

$$
\mathbf{E}^{\mathrm{i}}(\boldsymbol{r})=\left[1+\frac{\tau(\boldsymbol{r})}{3 j \omega \varepsilon_{0}}\right] \mathbf{E}(\boldsymbol{r})-P V \int_{V_{o b j}} \tau\left(\boldsymbol{r}^{\prime}\right) \overline{\mathbf{G}}\left(\boldsymbol{r}, \boldsymbol{r}^{\prime}\right) \cdot \mathbf{E}\left(\boldsymbol{r}^{\prime}\right) d v^{\prime}
$$

\section{Solution by 3D MoM}

This section presents a numerical solution of the integral equation (11) by 3D MoM. Firstly, we write the integral equation (11) in scalar form given by

$$
E_{x_{p}}^{\mathrm{i}}(\boldsymbol{r})=\left[1+\frac{\tau(\boldsymbol{r})}{3 j \omega \varepsilon_{0}}\right] E_{x_{p}}(\boldsymbol{r})-P V \int_{V_{o b j}} \tau\left(\boldsymbol{r}^{\prime}\right) \sum_{q=1}^{3} E_{x_{q}}\left(\boldsymbol{r}^{\prime}\right) G_{x_{p} x_{q}}\left(\boldsymbol{r}, \boldsymbol{r}^{\prime}\right) d v^{\prime}, p=q=1,2,3 .
$$

In this equation, we set $x_{1}=x, x_{2}=y$ and $x_{3}=z$. To solve the integral equation (12) by 3D MoM, we divide the volume $V_{o b j}$ into $N$ subvolumes $V_{m}(m=1, \ldots, N)$, where $E_{x_{p}}(\boldsymbol{r})$ and $\tau(\boldsymbol{r})$ are constant in each subvolume. With $\boldsymbol{r}_{m}$ as the point in the center of this subvolume, applying (12) to each subvolume $V_{m}$, we obtain 


$$
E_{x_{p}}^{\mathrm{i}}\left(\boldsymbol{r}_{m}\right)=\left[1+\frac{\tau\left(\boldsymbol{r}_{m}\right)}{3 \mathrm{j} \omega \varepsilon_{0}}\right] E_{x_{p}}\left(\boldsymbol{r}_{m}\right)-\sum_{q=1}^{3} \sum_{n=1}^{N}\left[\tau\left(\boldsymbol{r}_{n}\right) P V \int_{V_{\mathrm{n}}} G_{x_{p} x_{q}}\left(\boldsymbol{r}_{m}, \boldsymbol{r}^{\prime}\right) d v^{\prime}\right] E_{x_{q}}\left(\boldsymbol{r}_{n}\right)
$$

for $p, q=1,2,3$. The elements of $G_{x_{p} x_{q}}^{m n}$ are given by

$$
G_{x_{p} x_{q}}\left(\boldsymbol{r}, \boldsymbol{r}^{\prime}\right)=-j \omega \mu_{0}\left[\delta_{p q}+\frac{1}{k_{0}^{2}} \frac{\partial^{2}}{\partial x_{q} \partial x_{p}}\right] g_{0}\left(\boldsymbol{r}, \boldsymbol{r}^{\prime}\right), p, q=1,2,3
$$

An equivalent representation for (13) is

$$
\sum_{q=1}^{3} \sum_{n=1}^{N} G_{x_{p} x_{q}}^{m n} E_{x_{q}}\left(\boldsymbol{r}_{n}\right)=-E_{x_{p}}^{i}\left(\boldsymbol{r}_{m}\right), m=1 \ldots N, p, q=1,2,3
$$

This equation can be written as $[G][E]=-\left[E^{i}\right]$, where $[G]$ is a matrix of order $3 N \times 3 N$, while $[E]$ and $\left[E^{i}\right]$ are vectors of dimension $3 N$. The elements of $[G]$ are given by

$$
G_{x_{p} x_{q}}^{m n}=\tau\left(\boldsymbol{r}_{n}\right) P V \int_{V_{o b j}} G_{x_{p} x_{q}}\left(\boldsymbol{r}_{m}, \boldsymbol{r}^{\prime}\right) d v^{\prime}-\delta_{p q} \delta_{m n}\left[1+\frac{\tau\left(\boldsymbol{r}_{m}\right)}{3 j \omega \varepsilon_{0}}\right]
$$

where $\delta_{p q}=1$ if $p=q$ and $\delta_{p q}=0$ if $p \neq q ; \delta_{m n}=1$ if $m=n$ and $\delta_{m n}=0$ if $m \neq n$.

The total electric field at each point $\boldsymbol{r}_{m}$ is determined by inverting the matrix $[G]$. The elements of this matrix in (16) are calculated approximately by [19], [21]:

$$
G_{x_{p} x_{q}}^{m n}=\frac{-j \omega \mu_{0} k_{0} V_{n} \tau\left(\boldsymbol{r}_{n}\right) \exp \left(-j \alpha_{m n}\right)}{4 \pi \alpha_{m n}^{3}}\left[\left(\alpha_{m n}-1-j \alpha_{m n}\right) \delta_{p q}+\cos \theta_{x_{p}}^{m n} \cos \theta_{x_{q}}^{m n}\left(3-\alpha_{m n}^{2}+3 j \alpha_{m n}\right)\right]
$$

for $m \neq n$, and

$$
G_{x_{p} x_{q}}^{n n}=\delta_{p q}\left\{\frac{-2 j \omega \mu_{0} \tau\left(\boldsymbol{r}_{n}\right)}{3 k_{0}^{3}}\left[\left(1+j k_{0} a_{n}\right) \exp \left(-j k_{0} a_{n}\right)-1\right]-\left[1+\frac{\tau\left(\boldsymbol{r}_{n}\right)}{j \omega \varepsilon_{0}}\right]\right\}
$$

for $m=n$, where

$$
\begin{aligned}
& \boldsymbol{r}_{m}\left(x_{1}^{m}, x_{2}^{m}, x_{3}^{m}\right), \quad \boldsymbol{r}_{n}\left(x_{1}^{n}, x_{2}^{n}, x_{3}^{n}\right), \quad R_{m n}=\left|\boldsymbol{r}_{m}-\boldsymbol{r}_{n}\right|, \quad \alpha_{m n}=k_{0} R_{m n}, \quad a_{n}=\left(3 V_{n} / 4 \pi\right)^{1 / 3}, \\
& \cos \theta_{x_{q}}^{m n}=\frac{\left(x_{q}^{m}-x_{q}^{n}\right)}{R_{m n}}, \text { and } \quad \cos \theta_{x_{p}^{m n}}^{m}=\frac{\left(x_{p}^{m}-x_{p}^{n}\right)}{R_{m n}} .
\end{aligned}
$$


Obviously, the higher the number of subvolumes $N$ the better is approximation. The geometry of the subvolumes $V_{n}$ should be very close to a sphere of radius $a_{n}$. However, good results can be obtained using cubic cells as we show in this work.

After finding the electric field inside the volume $V_{o b j}$, one can calculate the electric field anywhere outside the nanoparticle by inversion of the system $[G][E]=-\left[E^{i}\right]$. To do this, we use the solution $[E]$ with (7) and (2) in points outside $V_{o b j}$.

\section{NUMERICAL RESULTS}

Based on the theoretical model presented in the previous section, we develop an algorithm in $\mathrm{C}$ language. To do this, we firstly define the constants and the variables of the problem. Then a 3D cubic domain of height and length $4 a$ is created, where $a=60 \mathrm{~nm}$ is the radius of the sphere. This spherical volume is divided into cubic cells of dimensions $d x=d y=d z$. The spherical volume with radius $a$ is created in the point $\left(x_{0}, y_{0}, z_{0}\right)$

$$
\sqrt{\left(x-x_{0}\right)^{2}+\left(y-y_{0}\right)^{2}+\left(z-z_{0}\right)^{2}}<a \text {. }
$$

The central sphere is positioned at the origin of coordinate axis $\left(x_{0}=y_{0}=z_{0}=0\right)$. For the case of the nanorod, we create a rectangular volume with the length $\mathrm{L}=60 \mathrm{~nm}$ and square cross section with the size $\mathrm{w}=10 \mathrm{~nm}$. The axis of the nanorod is placed along the axis $\mathrm{x}$, and it is centered at the origin of the coordinate system.

The method does not require an Absorbing Boundary Conditions (ABCs) to simulate a free space radiation because the Green's function already takes into account the radiation condition. The ABCs are used in other numerical methods, such as the FDTD or professional programs such as software Comsol Multiphysics. The latter uses the Finite Element Method (FEM) and a special type of ABC, known as perfectly matched layer (PML), or radiation condition, for an artificial absorption of electromagnetic waves.

All the cells created inside the object are excited by a plane wave $\left[E^{i}\right]$. Subsequently, the elements of the matrix $[G]_{3 N X 3 N}$ are calculated for the volume of the object using (17) and (18) for $m \neq n$ and $m=n$, respectively. The field inside the volume $V_{o b j}$ is obtained by the inversion of the linear system in the form $[E]=-[G]^{-1}\left[E^{i}\right]$. This linear system is solved by the Gaussian elimination method for complex numbers. Then we calculate the total field in any point outside the volume $V_{o b j}$ with (2) and with the fields inside $V_{o b j}$ calculated in the previous step.

\section{A. Nanosphere}

Initially, for validation of the algorithm we compare our results with the classical solution for the sphere by analytical Mie model [18]. We also compare our results with those obtained by the software 
Comsol Multiphysics. Subsequently, also we analyze a rectangular nanorod to confirm the possibility of implementing the algorithm for different geometries. All the simulations were realized in a core i7 computer with $16 \mathrm{G}$ of RAM.

The analyzed nanosphere has the radius $a=60 \mathrm{~nm}$. In this case, we use the total number of cubic elements $N=1791$ with the dimensions $d x=d y=d z=8 \times 10^{-9}$. The results of calculations for the near fields in the axis $x, y$ and $z$ are shown in Figs. 3-5.The amplitudes of these fields are normalized to the magnitude of the incident plane wave $\mathrm{E}_{\mathrm{i} 0}$. The electromagnetic wavelength used in all simulations is $\lambda=550 \mathrm{~nm}$, which is near the resonance.

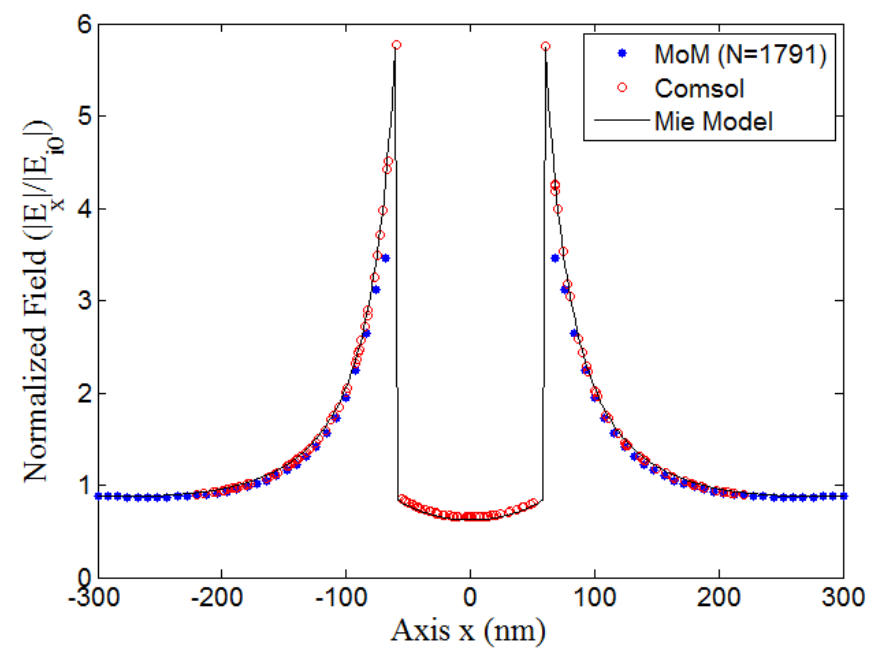

Fig.3. Normalized near field distribution for $\lambda=550 \mathrm{~nm}$ along axis $x$ for gold sphere with radius $a=60 \mathrm{~nm}$.

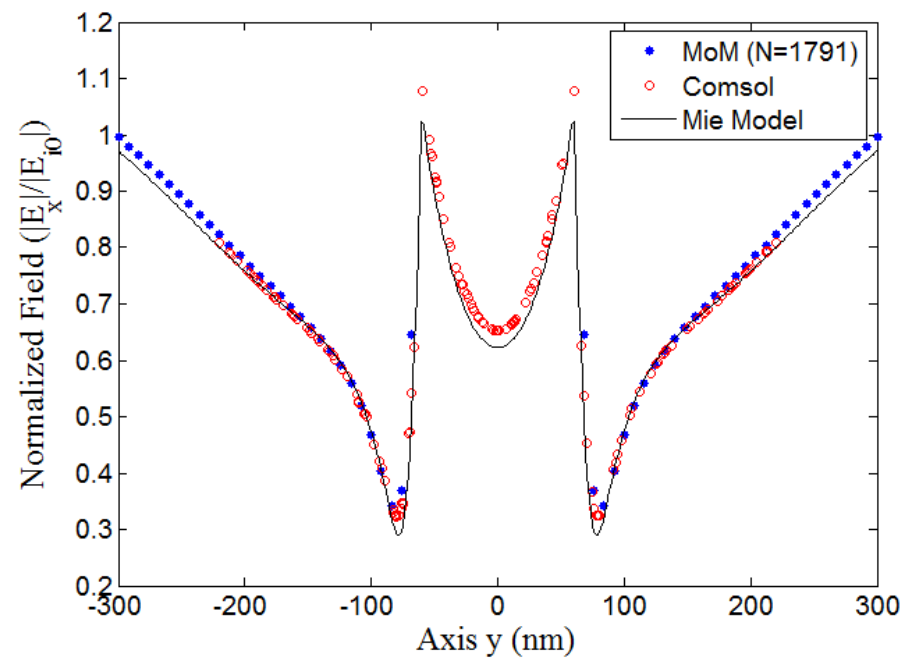

Fig.4. Normalized near field distribution for $\lambda=550 \mathrm{~nm}$ along axis $y$ for gold sphere with radius $a=60 \mathrm{~nm}$. 


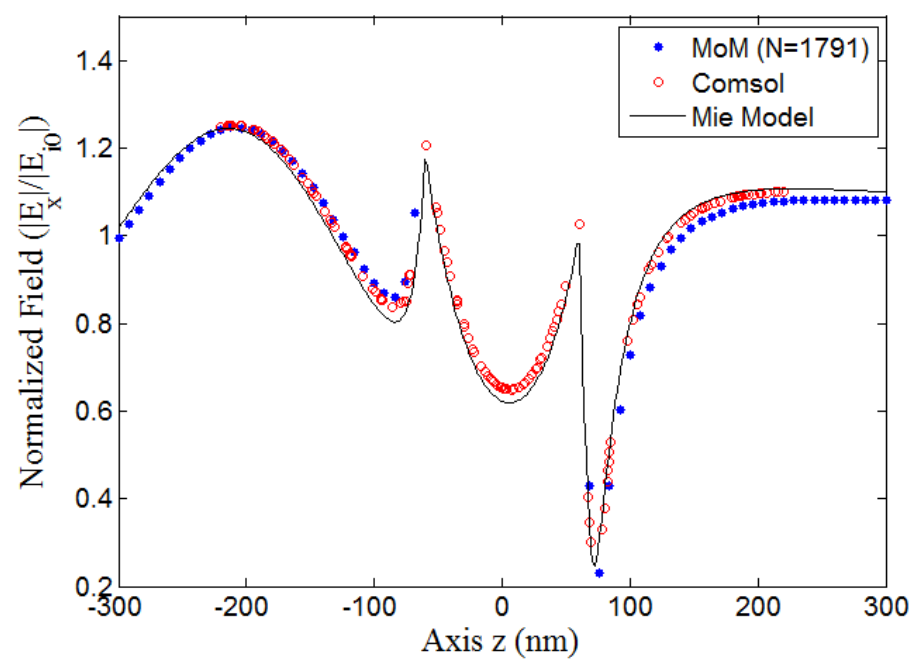

Fig.5. Normalized near field distribution for $\lambda=550 \mathrm{~nm}$ along axis $z$ for gold sphere with radius $a=60 \mathrm{~nm}$.

In Comsol simulation, we created a mesh of 131638 elements inside a spherical domain with diameter $\mathrm{D}=460 \mathrm{~nm}$. The spherical domain is limited by a PML absorbing boundary condition to simulate the open space. In Figs. 3-5, we observe a good agreement between the results for the used discretizations. However, for the points near the surface of the sphere one can note a certain difference in the results. This can be explained by rapid variation of the field in this region. This means that we need a finer discretization in these regions to obtain a higher accuracy.

Fig.6 shows the variation of the x component of the normalized electric field versus wavelength for different points along the axis $\mathrm{x}$. The range of the analyzed wavelengths is between $500 \mathrm{~nm}$ and $1000 \mathrm{~nm}$. The resonance of the sphere occurs near $\lambda=550 \mathrm{~nm}$. The points where the electric fields were calculated are positioned in $(x=a+d, y=0, z=0), a$ is the radius of the sphere, and $d$ runs through the values $20 \mathrm{~nm}, 40 \mathrm{~nm}, 80 \mathrm{~nm}$ and $160 \mathrm{~nm}$.

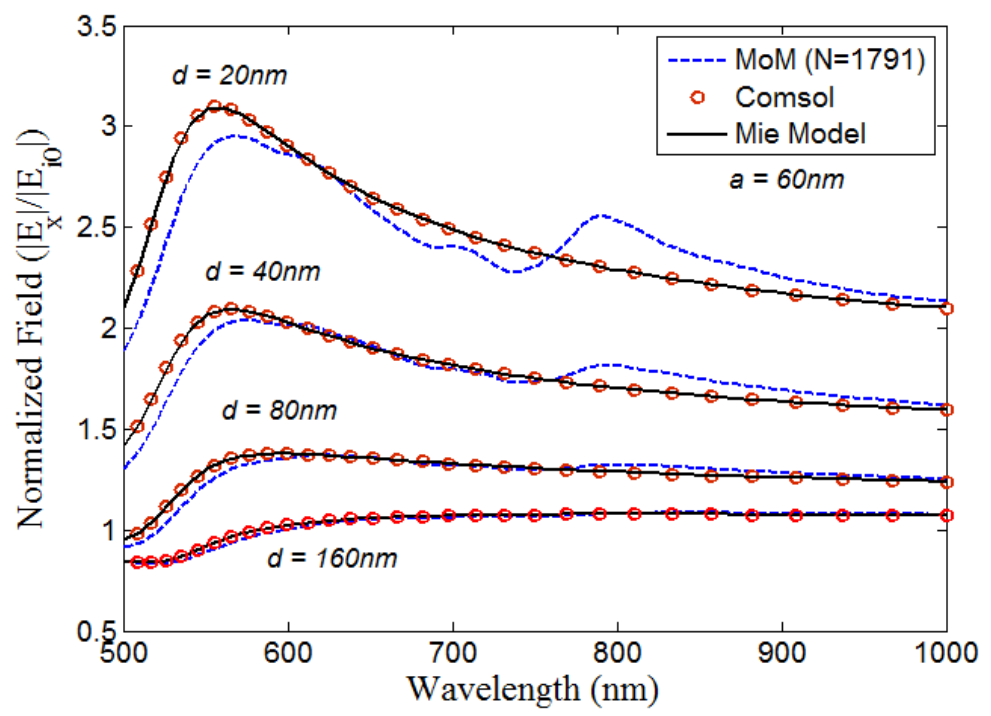

Fig. 6. Spectral response of normalized electric field (x component) near nanosphere in different points along axis $\mathrm{x}(x=a+d, y=0, z=0)$, $a=60 \mathrm{~nm}, d=20,40,80$, and $160 \mathrm{~nm}$. 
We observe from this figure a good agreement between the calculated results. For the points near the sphere, electromagnetic fields vary rapidly with distance, therefore, it is necessary a finer discretization of the nanosphere, as it was observed also in Figs. 3-5.

\section{B. Rectangular Nanorod}

The analyzed rectangular nanorod has length $\mathrm{L}=60 \mathrm{~nm}$, and width $\mathrm{w}=10 \mathrm{~nm}$. For this case, we performed two simulations with different number of total cubic cells elements of the discretization $N$ to estimate convergence of the method. In one simulation, we used $N=750$, cubic cells of dimensions $d x=d y=d z=2 \times 10^{-9}$, and in the other one we used $N=6000$, with cubic cells of dimensions $d x=d y=d z=1 \times 10^{-9}$. We also simulated this nanorod using Comsol software. In the latter case we used a mesh with 371747 elements, and a spherical domain limited by a PML. All the simulations were realized in a core i7 computer with $16 \mathrm{G}$ of RAM.

We show in Fig. 7 the normalized electric field $\mathrm{E}_{\mathrm{x}}$ at the end of nanorod at the point $(x=\mathrm{L} / 2+\mathrm{d}, y$ $=0, z=0$ ), where $\mathrm{d}=5 \mathrm{~nm}$ and $20 \mathrm{~nm}$. Figs. 8-10 show the spectral response of the normalized electric field in the axis $\mathrm{z}$ near the middle of the nanorod at the points $(0,0, \mathrm{w} / 2+\mathrm{d})$, where $\mathrm{w}=10 \mathrm{~nm}$ is the side of the square cross section of the nanorod (Fig. 1b), and d =5nm, 10nm and 20nm, respectively. The wavelength interval extends from $500 \mathrm{~nm}$ to $1300 \mathrm{~nm}$, where the principal resonances of the nanorod occur.

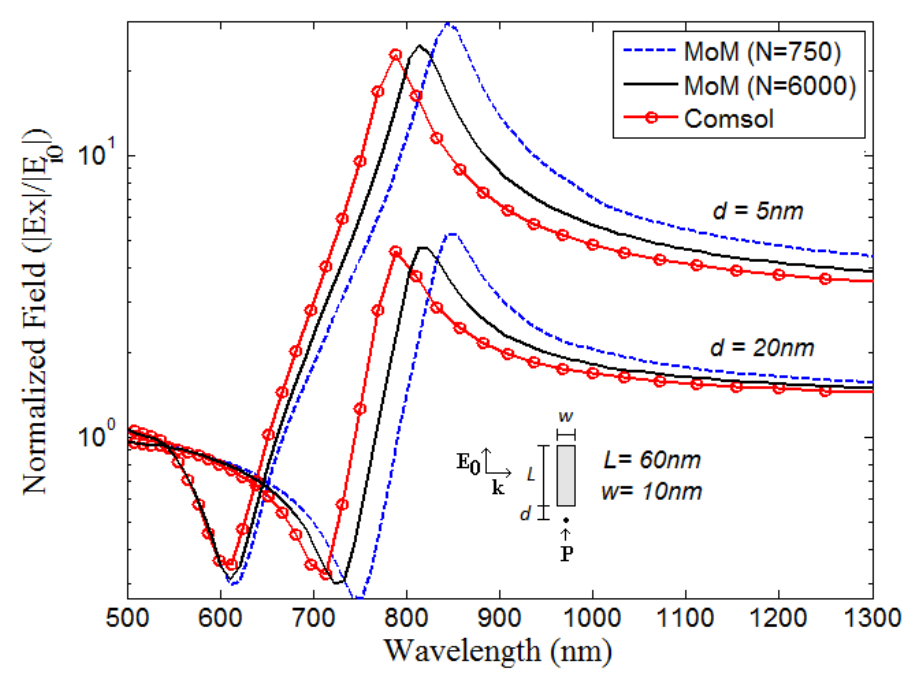

Fig. 7. Spectral response of normalized electric field (x component) near edge of nanorod in different points along the axis $\mathrm{x}$ at points $(x=\mathrm{L} / 2+d, y=0, z=0), \mathrm{L}=60 \mathrm{~nm}, \mathrm{w}=10 \mathrm{~nm}, \mathrm{~d}=5,20 \mathrm{~nm}$. 


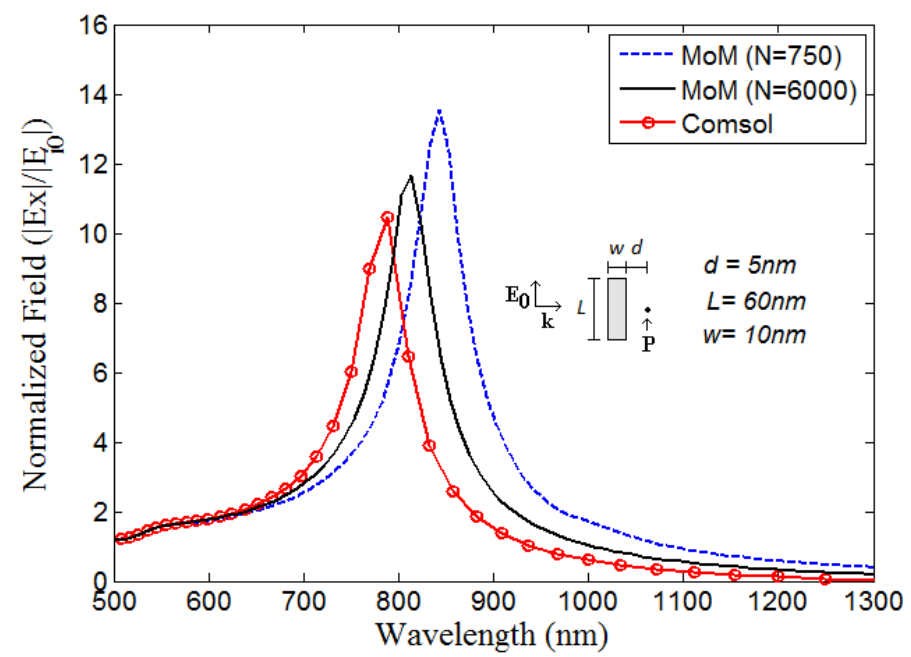

Fig. 8. Spectral response of normalized electric field (x component) near middle of nanorod at point $(x=0, y=0, z=\mathrm{w} / 2+d)$ with $\mathrm{L}=60 \mathrm{~nm}$, $\mathrm{w}=10 \mathrm{~nm}, \mathrm{~d}=5 \mathrm{~nm}$.

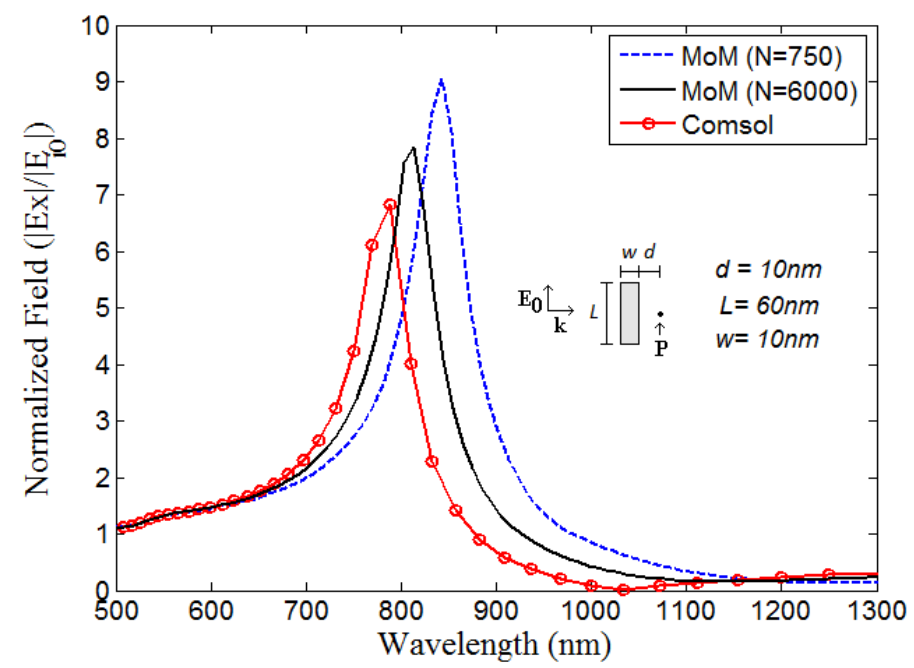

Fig. 9. Spectral response of normalized electric field (x component) near middle of nanorod at point $(x=0, y=0, z=\mathrm{w} / 2+d), \mathrm{L}=60 \mathrm{~nm}, \mathrm{w}=$ $10 \mathrm{~nm}, \mathrm{~d}=10 \mathrm{~nm}$.

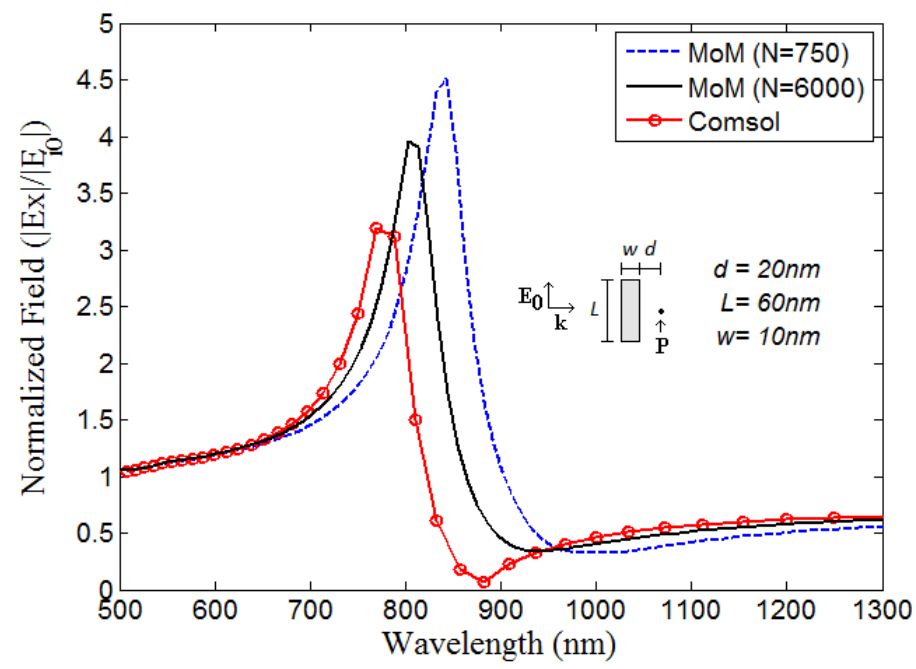

Fig. 10. Spectral response of normalized electric field (x component) near center of nanorod at point $(x=0, y=0, z=\mathrm{w} / 2+d), \mathrm{L}=60 \mathrm{~nm}$, $\mathrm{w}=10 \mathrm{~nm}, \mathrm{~d}=20 \mathrm{~nm}$. 
We observe from Figs. 7-10 a good agreement between the MoM and Comsol Multiphysics results. These figures show that with increase of $N$, our results converge to the Comsol simulation.

\section{CONCLUSIONS}

We presented in this paper a 3D MoM computational algorithm for efficient full-wave electromagnetic scattering analysis of plasmonic nanostructures. The method was codified in $\mathrm{C}$ language, and two gold nanoparticles were analyzed: nanosphere and rectangular nanorod. To validate the computational code, we compared our results with simulations carried out by the commercial package Comsol. In case of nanosphere, we also compared the obtained results with the classical analytical Mie model. In all cases, we observed a good agreement between the results obtained by our code, the analytical model and the commercial software. We also analyzed convergence of the method. For this purpose, we used different number of cubic cells $N$. For large values of $N$, our results approach to the Comsol simulation. The full wave method is quite general and can be used to analyze plasmonic nanostructures with different geometries and excitation sources. However, the method requires a good computational capacity in terms of memory and processing speed.

\section{ACKNOWLEDGMENT}

This work was financially supported by the Amazon Foundation of Research of the State of ParáFapespa - Brazil.

\section{REFERENCES}

[1] M. L. Brongersman and P. G. Kik, Surface Plasmon Nanophotonics, Netherlands: Springer, 2007.

[2] L. Novotny, and B. Hecht, Principles of Nano-Optics, New York: Cambridge, 2006.

[3] N. C. Lindquist, et al, "Engineering metallic nanostructures for plasmonics and nanophotonics", Rep. Prog. Phys., vol. 75, p. 036501, 2012.

[4] O. Sqalli, I. Utke, P. Hoffmann and F. M.-Weible, "Gold elliptical nanoantennas as probes for near field optical microscopy," J. of Appl. Physics, vol. 92, pp. 1078-1083, July 2002.

[5] D. W. Pohl, "Near field optics as an antenna problem"', Near Field: Principles and Applications, The second Asia-Pacific Workshop on Near Field Optics, Beijing, China October 20-23, pp. 9-21, 1999.

[6] K. Q. Costa, and V. Dmitriev, "Analysis of modified bowtie nanoantennas in the excitation and emission regimes," Prog. in Electro. Research B, vol. 32, p. 57-73, 2011.

[7] P. Bharadwaj, et. al., "Optical antennas", Adv. in Opt. and Photon., vol. 1, pp. 438-483, Aug. 2009.

[8] L. Novotny and N. V. Hulst, "Antennas for light", Nature Photon., vol. 5, 2011.

[9] P. Biagioni, J.-S. Huang, and B. Hecht, "Nanoantennas for visible and infrared radiation”, Rep. Prog. Phys., vol. 75, p. 024402, 2012.

[10] H. Wang, C. T. Chong and L. Shi, "Optical antennas and their potential applications to 10 terabit/in ${ }^{2}$ recording," Optical Data Storage Topical Meeting, pp. 16-18, May 2009.

[11] T. A. El-Brolossy, T.Addallah, M.B. Mohamed, S. Abdallah, K. Easawi, S. Negm and H. Talaat, "Shape and size dependence of the surface plasmon resonance of gold nanoparticles studied by photoacoustic technique," The European Physical Journal Special Topics, vol. 153, pp. 361-364, 2008.

[12] C. Hrelescu, T.K.Sau, A.L.Rogach, Frank Jackel and J. Feldmann, "Single gold nanostars enhance Raman scattering," Applied Physics Letters, vol. 94, 153113, 2009.

[13] D. Cho, F. Wang, J. Valentine, Z. Yu, Y. Liu, X. Zhang and Y.R. Shen, "Plasmon Resonances of strongly coupled nanodisks," NanoOptoelectronics Workshop, pp. 88-89, 2007.

[14] V. Germain, A. Brioude, D. Ingert, and M. P. Pileni, "Silver nanodisks: size selection via centrifugation process and optical properties," The Journal of Chemical Physics, vol. 122, 124707, 2005.

[15] W. Rechgerger, A. Hohenau, A. Leitner, J. R. Krenn, B. Lamprecht, F. R. Aussenegg, "Optical properties of two interacting gold nanoparticles,"Optics Communications, vol. 220, pp. 137-141, 2003.

[16] J. Neleyah, M. Kociaak, O. Sthepan, F. J. G. de Abajo, M. Tence, L. Henrard, D. Taverna, I. Pastoriza-Santos, L. M. Liz-Marza and C. Colliex, "Mapping surfaceplasmons on a single metallic nanoparticle," Nature Physics, vol. 3, pp. 2248-353, 2007.

[17] D. E. Livesay, and K. M. Chen, "Electromagnetic fields induced inside arbitrary shaped biological bodies", IEEE Trans. Micro. Theo. Tech., 22(12): pp. 1273-1280, 1974.

[18] J. A. Stratton, Electromagnetic Theory, New York: McGraw-Hill, 1941.

[19] C. A. Balanis, Advanced Engineering Electromagnetics,Jonh Wiley \&Sons , pp.327-328, 1989.

[20] J. Van Bladel, “Some remarks on Grenns's dyadic for infinite space,” IRE transactions on Antennas and Propagation, vol 9, pp. 563$566,1991$.

[21] M. N.O.Sadiku, “Numerical Techniques in Electromagnetics," Second Edition CRC: pp. 350-360, 2001. 